\title{
RESTAURACIÓN DE BOSQUES EN TERRITORIOS INDÍGENAS DE CHIAPAS: MODELOS ECOLÓGICOS Y ESTRATEGIAS DE ACCIÓN
}

\author{
Mario González-Espinosa' ${ }^{1,7}$, Neptalí Ramírez-Marcial ${ }^{1}$, AnGélica Camacho-CruZz, ${ }^{2,}$, \\ Silvia C. Holz ${ }^{4}$, José María Rey-Benayas ${ }^{5}$ y Manuel R. Parra-Vázquez ${ }^{6}$ \\ 'El Colegio de la Frontera Sur (ECOSUR), División de Conservación de la Biodiversidad, Departamento de Ecología y \\ Sistemática Terrestres, San Cristóbal de Las Casas 29290, Chiapas, México. \\ ${ }^{2}$ Universidad Complutense, Departamento Interuniversitario de Ecología, Madrid 28040, España. \\ ${ }^{3}$ Biodiversidad: Conservación y Restauración, A.C. (BIOCORES), Tapachula 17, \\ Barrio El Cerrillo, San Cristóbal de Las Casas 29220, Chiapas, México. \\ ${ }^{4}$ Universidad de Buenos Aires, Departamento de Ecología, Genética y Evolución, Buenos Aires 1428, Argentina. \\ ${ }^{5}$ Universidad de Alcalá, Departamento Interuniversitario de Ecología, Alcalá de Henares 28871, España. \\ ${ }^{6}$ El Colegio de la Frontera Sur (ECOSUR), División de Sistemas de Producción Alternativos, Departamento de Gestión \\ de los Recursos Naturales, San Cristóbal de Las Casas 29290, Chiapas, México. \\ 7Autor para la correspondencia. Tel +52 967678 4558, 529676749000 ext. 1318, \\ Fax +52 967678 4557; correo-e: mgonzale@sclc.ecosur.mx
}

\begin{abstract}
Resumen: Los bosques de las montañas del sur de México han sido fragmentados y alterados en su estructura y composición florística por agricultura tradicional asociada al crecimiento poblacional. La restauración forestal no sólo es urgentemente necesaria en muchas áreas de Chiapas, sino que es una opción viable para recuperar servicios y productos que en adelante pueden utilizarse de manera sustentable. La restauración de los bosques debe partir del entendimiento de los procesos ecológicos básicos, para luego diseñar las medidas técnicas pertinentes e incorporar elementos económicos y sociales que guíen un enfoque adaptativo de los programas de acción. Al cabo de más de una década, nuestras experiencias de investigación, desarrollo tecnológico y vinculación social permiten proponer modelos que abarcan algunos de los aspectos ecológicos, técnicos y sociales implicados en la recuperación, conservación y aprovechamiento racional de los recursos forestales.
\end{abstract}

Palabras clave: bosques de montaña, fragmentación, gradientes, riqueza florística, vinculación social.

\begin{abstract}
Montane forests of southern Mexico have been severely fragmented and their structure and floristic composition have been altered because of traditional agriculture and population growth. In extensive areas of Chiapas, forest restoration is not only an urgent need, but it also represents a viable option for the recovery of products and services that could be used in the future in a sustainable way. Forest restoration should be based upon the understanding of basic ecological processes followed by the design of appropriate practices, and taking into account economic and social issues that may guide an adaptive approach of the action programs. Research, development, and outreach experiences spanning more than a decade lead us to suggest models for some of the ecological, technical, and social issues underlying the recovery, conservation, and rational use of forest resources.
\end{abstract}

Key words: floristic richness, fragmentation, gradients, montane forests, outreach.

L as montañas del sur de México albergan una gran variedad de formaciones forestales con una elevada riqueza de especies (Miranda, 1952; Breedlove, 1981; GonzálezEspinosa et al., 2004, 2005b). En décadas recientes su extensión ha sido drásticamente reducida por la ampliación de la agricultura tradicional, asociada al crecimiento poblacional, la globalización y la aspiración de mejores niveles de vida (Parra-Vázquez y Díaz-Hernández, 1997; Montoya-Gómez et al., 2003; Villafuerte-Solís, 2004). De manera similar a lo observado en otras regiones del mundo con alta riqueza biológica (Mace y Pagel, 1995; Moore et al., 2002), en Chiapas la biodiversidad se corresponde con el acervo cultural (Berlin et al., 1974; Perales et al., 2005). La distribución de los bosques coincide a menudo con los 
límites territoriales de comunidades indígenas de varias etnias (zoques, tzotziles, tzeltales, tojolabales, choles y lacandones) y la apropiación de sus territorios con frecuencia ha promovido la fragmentación de los bosques (Ixtacuy-López et al., 2006). A pesar del predominio de las áreas de vegetación secundaria y de agricultura, notablemente más pobres que los bosques originales en su estructura y diversidad (Quintana-Ascencio et al., 1996; MontesAvelar, 2001), las comunidades indígenas obtienen de los fragmentos forestales una considerable variedad de recursos (Collier, 1976; Hellier et al., 1999; Levy-Tacher et al., 2002).

No se conocen los valores ni los usos que pueden alcanzar en el mercado los productos de las numerosas especies utilizadas por los grupos indígenas de Chiapas. Con todo, parece adecuado buscar el uso de una alta diversidad de especies, segregadas en grupos funcionales, en la restauración de los bosques y selvas basada en modelos ecológicos de la organización de la diversidad arbórea durante la sucesión (Bradshaw, 1987, 2002; Ramírez-Marcial et al., 2005). La diversificación de los bosques puede favorecer la estabilidad productiva y económica, a diferencia de las plantaciones monoespecíficas, frecuentemente de especies exóticas, que pueden provocar impactos ambientales indeseables. La restauración de rodales con alta diversidad puede ofrecer un potencial de beneficios a la conservación de la biodiversidad local, incluso si los fragmentos iniciales son relativamente pequeños (Sayer et al., 2004).

En este trabajo presentamos una revisión de elementos básicos que han servido para configurar algunos modelos ecológicos y de acción institucional, particulamente incipientes estos últimos, que pueden sustentar aplicaciones en restauración de bosques en el contexto particular de Chiapas. A partir de la imitación de la estructura, la composición y el funcionamiento sostenible de los sistemas naturales, se busca evaluar la salud ambiental junto con la viabilidad económica en la explotación del bosque. En una etapa avanzada del proceso de restauración se busca incidir en valores más amplios de la sociedad: respeto y aliento de la cultura autóctona y la universal, bienestar social, tolerancia y convivencia política en la paz, goce estético y conciencia histórica, entre otros (Higgs, 1997; Cairns, 2002). Para nuestro caso, los aspectos operativos de la práctica de la restauración implican también definir posiciones y estrategias de acción de los grupos académicos en el contexto de redes para la cooperación técnica y el financiamiento con organizaciones de diversa índole en ámbitos variados (Lyall et al., 2004; Guimerà et al., 2005). Nuestra experiencia incluye en su mayor parte el desarrollo de investigación ecológica básica y aplicada a la restauración que hemos realizado por más de un decenio en dos regiones de Chiapas, Los Altos y las Montañas del Norte. Sin embargo, nuestra intención es, a partir de estos casos, que abarcan una considerable variedad de condiciones ecológi- cas, mostrar un conjunto de modelos dirigidos a la restauración para su aplicación en otras condiciones naturales.

\section{Escenario ecológico y socioeconómico}

Riqueza florística. La amplitud latitudinal de Chiapas, su orografía y su historia geológica determinan una amplia variedad de condiciones ecológicas y una notable diversidad biológica (Miranda, 1952; Breedlove, 1981, 1986; González-Espinosa et al., 2005a). Dentro de los límites

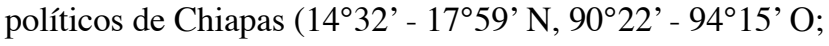
$75,634 \mathrm{~km}^{2}$ ) se reconocen siete regiones fisiográficas y se estima una riqueza florística de $c a$. 10,000 especies de plantas vasculares (D. E. Breedlove, com.pers.). A nivel nacional esta flora es una de las más diversas y contribuye a que México tenga un reconocimiento internacional en esta materia (Rzedowski, 1993; Villaseñor, 2003; Villaseñor et al., 2005). Se reconocen en Chiapas entre 12 y 18 formaciones vegetales, de acuerdo con Miranda (1952) y Breedlove (1981), respectivamente. De manera conservadora, la riqueza de especies arbóreas en Chiapas incluye 1,400 - 1,500 taxa (González-Espinosa et al., 2004, 2005b).

En Chiapas hay cerca de 15,000 $\mathrm{km}^{2}$ de asociaciones de bosque de pino, bosque de pino-encino y bosque mesófilo de montaña (Palacio-Prieto et al., 2000) que constituyen la vegetación predominante en la Sierra Madre, Los Altos y las Montañas de Norte. Las especies de Pinus y Quercus dominan el dosel de diversas asociaciones localizadas por encima de $900 \mathrm{~m}$ de altitud. La riqueza arbórea regional (diversidad gamma) de estas formaciones es muy alta (cuadro 1), aunque ocasionalmente pueda haber sólo 10 15 especies de árboles a escala local (diversidad alfa). A su vez, las ramas y los troncos de los encinos de gran tamaño pueden soportar una gran diversidad de epífitas vasculares (helechos, orquídeas y bromeliáceas; Wolf y Flamenco, 2003, 2005). El alcance de la restauración, asumida como un reto que finalmente se aplica en escalas espaciales de paisaje y región, implica un potencial florístico considerabl e dada la mezcla de especies de diferente condición sucesional y afinidad fitogeográfica (Ramírez-Marcial et al., 2005; cuadro 1).

Deforestación reciente. En las regiones altas de Chiapas se ha mantenido durante los últimos 50 años una tendencia hacia la fragmentación de los bosques maduros y su sustitución por áreas de cultivo y comunidades secundarias asociadas a la agricultura de milpa (Wagner, 1962; GonzálezEspinosa et al., 1991; Ramírez-Marcial et al., 2001). El deterioro más acelerado de los bosques en Chiapas se inició en la década de los setentas. Paradójicamente, medidas conservacionistas decretadas de manera autoritaria, como la suspensión de aprovechamientos forestales en Chiapas a partir de 1991 ("veda forestal"), que coincidieron o incluso 
Cuadro 1. Riqueza conocida de especies de Quercus, Pinus y otras especies arbóreas latifoliadas (agrupadas de acuerdo con su estatus sucesional como tempranas, intermedias y tardías), en intervalos de altitud de $500 \mathrm{~m}$ en bosques de montaña de Chiapas. Basado en los ejemplares de herbario depositados en CAS, DS, MEXU y ECOSUR (y parcialmente en ENCB, XAL y CHAPA) recolectados entre 1865 y 1999 . Asignación sucesional de acuerdo con González-Espinosa et al. (2005b). Las especies de Quercus han sido nombradas con base en Breedlove (1986) y una lista no publicada proporcionada por D. E. Breedlove (com.pers.); las otras especies latifoliadas con base principalmente en Breedlove (1986); las de Pinus de acuerdo con Farjon y Styles (1997). Ver detalles de la base de datos de ejemplares de herbario utilizada en GonzálezEspinosa et al. (2004, 2005b).

\begin{tabular}{|c|c|c|c|c|c|}
\hline \multirow[t]{2}{*}{ Grupos ecológicos } & \multicolumn{5}{|c|}{ Altitud (m) } \\
\hline & $500-999$ & $1,000-1,499$ & $1,500-1,999$ & $2,000-2,499$ & $2,500-3,000$ \\
\hline Especies de Pinus & 5 & 6 & 10 & 11 & 4 \\
\hline Especies de Quercus & 17 & 24 & 23 & 20 & 8 \\
\hline Total de especies de Pinus y Quercus & 22 & 30 & 33 & 31 & 12 \\
\hline \multicolumn{6}{|l|}{ Especies distintas de Pinus y Quercus } \\
\hline Sucesionales tempranas & 103 & 163 & 158 & 88 & 33 \\
\hline Sucesionales intermedias & 117 & 154 & 149 & 132 & 37 \\
\hline Sucesionales tardías & 24 & 36 & 71 & 68 & 31 \\
\hline Subtotal & 244 & 353 & 378 & 288 & 101 \\
\hline Riqueza total conocida de árboles & 266 & 383 & 411 & 319 & 113 \\
\hline
\end{tabular}

contribuyeron al inicio del conflicto armado de 1994, aceleraron los procesos de deterioro generalizado de los recursos forestales y los cambios de uso del suelo que se observaron durante casi todo el decenio (Collier y Quaratiello, 1994; González-Espinosa, 2005; IxtacuyLópez et al., 2006). Se ha estimado para los últimos 35 años una pérdida de más de la mitad del área original de bosques en el centro de Chiapas. Entre 1970 y 1990 el área de "bosques cerrados" en Los Altos del centro de Chiapas se redujo de 256,560 a 125,949 ha (una reducción de 49\%), mientras que la superficie de bosques fragmentados y/o degradados incrementó de 203,236 a 316,414 ha (64\%) y la de tierras abiertas para usos agropecuarios aumentó de 159,785 a 177,248 ha (9\%; ver detalles en de Jong et al., 1999). Las tasas anuales de deforestación han sido muy variables según los municipios y la duración del período considerado, pero en general, desde 1974 han superado a la media anual nacional $(1.6 \%)$ y en algunos lugares de Los Altos han alcanzado valores tan elevados como $4.9 \%$ (Ochoa-Gaona y González-Espinosa, 2000). Un estudio reciente estimó una tasa anual de deforestación de más de $6 \%$ para el período que comprende los años inmediatamente siguientes al levantamiento zapatista de 1994 (Cayuela et al., 2005, 2006).

Empobrecimiento florístico y funcional de los bosques. Junto con la deforestación, la fragmentación y el aislamiento de los rodales, se ha producido un empobrecimiento florístico de los bosques secundarios (Ramírez-Marcial et al., 2001; Ochoa-Gaona et al., 2004). La tendencia observada en bosques previamente dominados por especies de
Quercus es el reciente incremento de Pinus spp., asociado con una reducción en la riqueza de bejucos, lianas, arbustos y árboles del interior (González-Espinosa et al., 1995). El deterioro se puede relacionar con factores sociales como el incremento de la densidad poblacional y la creación de nuevos asentamientos (Ixtacuy-López et al., 2006), la emigración, los niveles de pobreza, la adopción de nuevos cultivos como la cafeticultura (Ixtacuy-López y ParraVázquez, 2005), el desarrollo de infraestructura y los mayores riesgos de incendios extensos en años muy secos (Román-Cuesta et al., 2003). El estudio de las consecuencias funcionales de la deforestación, la fragmentación y el empobrecimiento de la riqueza de los bosques del sur de México es una tarea apenas iniciada. Sin embargo, la información disponible indica que los pinares inducidos varían más en su temperatura y humedad interior, y que sus suelos son más compactos y menos fértiles (Romero-Nájera, 2000; Galindo-Jaimes et al., 2002; García-Barrios y González-Espinosa, 2004). Estos cambios pueden comprometer, a su vez, la regeneración de numerosas especies arbóreas asociadas a los ambientes umbrosos y húmedos bajo doseles cerrados de Quercus spp. (Galindo-Jaimes et al., 2002; Ramírez-Marcial, 2003; Asbjornsen et al., 2004).

Crecimiento poblacional e inercia social. El sur de México incluye algunas de las regiones con menores índices de desarrollo económico y bienestar social, junto con algunas de las mayores tasas de crecimiento poblacional (MontoyaGómez et al., 2003; Vásquez-Sánchez, 2003). El potencial de sustento local es relativamente limitado, ya que los suelos (en su mayoría rendzinas, litosoles, regosoles y luvi- 
soles) son poco aptos para la agricultura (VásquezSánchez, 2003). Sin embargo, el paisaje tiene potencial para el aprovechamiento forestal y el nivel de organización política necesaria de muchas comunidades es elevado (Cartagena-Ticona et al., 2005; Bojórquez-Vargas, 2006). No obstante, prevalecen, junto con la ausencia de financiamiento, situaciones derivadas del minifundismo, del régimen de propiedad de la tierra y de la frágil convivencia política que dificultan la consolidación de áreas para uso forestal a una escala industrial. Aunque en la región de estudio se tienen niveles bajos o medios de riesgo de conflictos por el uso del territorio, persisten algunas áreas con mayor volatilidad social (e.g. los municipios de Simojovel, Chenalhó, San Juan Chamula y San Andrés Larráinzar). Recientemente se han sistematizado experiencias de algunas comunidades rurales mayas que muestran elementos valiosos en el aprovechamiento de los recursos forestales: las formas comunales de gobierno (Holder, 2004; Bojórquez-Vargas, 2006), las responsabilidades compartidas y descentralizadas sobre el territorio y la capacidad organizativa para desarrollar empresas comunitarias (Bray y Merino, 2004; Castillo et al., 2005). En la región que nos ocupa, como en otras de América Latina, se han dado avances para apoyar el desarrollo comunitario tales como la formación de los consejos de desarrollo rural sustentable en los órdenes de gobierno federal, estatal y municipal. Aunque aún muestran un desempeño incipiente, los consejos representan espacios potenciales para el debate y la toma de decisiones sobre el manejo de los recursos naturales (Cartagena-Ticona et al., 2005). Con todo, prevalecen condiciones políticas, sociales y económicas que limitan la posibilidad de logros como los de otras comunidades también poseedoras de bosques de pino-encino en Oaxaca, Michoacán y Puebla (Bray y Merino, 2004).

\section{Conceptos ecológicos y avances}

Sucesión secundaria. La restauración de ecosistemas forestales aspira a simular, e incluso acelerar, los procesos que dirigen la sucesión secundaria en comunidades arboladas. La información requerida para predecir las tendencias de cambio sucesional en los bosques de montaña del sur de México es considerable pero insuficiente. Se conocen algunos de los principales rasgos estructurales, florísticos y edáficos de la secuencia sucesional derivada de la agricultura tradicional (González-Espinosa et al., 1991, 2006; Luna et al., 2001; Negrete-Yankelevich, 2004; Bautista-Cruz et al., 2005). Las etapas sucesionales avanzadas incluyen en el dosel una mezcla de especies de amplia distribución y afinidad holártica, junto con algunas pocas que son ra ras o escasas (Quintana-Ascencio y González-Espinosa, 1993; Ramírez-Marcial, 2003). Las etapas sucesionales de diferentes tipos de bosques, desde pinares hasta bosques de neblina, comparen muchas especies (González-Espinosa et al., 2006); este atributo regional permite la restauración con un número alto, pero manejable, de especies (Ramírez-Marcial et al., 2005).

Se ha identificado a la facilitación y la tolerancia como determinantes del cambio en composición y abundancia en etapas iniciales de la sucesión. Los individuos de Baccharis vaccinioides, un arbusto dominante de los matorrales que se establecen en campos agrícolas abandonados, ejercen un efecto de nodrizas sobre las plántulas de Quercus spp. y Pinus que con el tiempo formarán el dosel de los acahuales e incluso de los bosques maduros (Ramírez-Marcial et al., 1996). Al cabo de ocho años se ha encontrado que Abies guatemalensis, Pinus ayacahuite y $P$. pseudostrobus pueden sobrevivir y crecer mejor en áreas abiertas, de manera contraria a varias especies arbóreas latifoliadas que lo hicieron mejor bajo doseles cerrados (QuintanaAscencio et al., 2004). Se han estudiado los efectos de la composición del dosel y el mantillo sobre el establecimiento temprano de varias especies. Por ejemplo, las semillas de Cornus disciflora tienen menor germinación en pinares que en encinares; la supervivencia de Drimys granadensis y Quercus laurina es máxima en bosques maduros de encino y en pinares, respectivamente (Camacho-Cruz et al., 2000). El paisaje agrícola y forestal creado por el patrón regional de uso del suelo determina interacciones de depredación y dispersión de semillas de las especies dominantes de Quercus (López-Barrera et al., 2005, 2006, 2007), que a su vez determinan las condiciones de regeneración para otras especies latifoliadas (Camacho-Cruz et al., 2000).

Ecología del disturbio antrópico. El disturbio crónico, de baja intensidad pero mantenido durante largos periodos, es un resultado típico del aprovechamiento indígena tradicional de los recursos forestales (Ramírez-Marcial et al., 2001; Martorell y Peters, 2005). No obstante su relevancia, ha sido un área de trabajo relativamente desdeñada por la comunidad ecológica, que se ha orientado más al estudio de los disturbios de origen natural (a menudo de carácter catastrófico como los deslaves, las erupciones volcánicas, los incendios y los huracanes, aunque también aquellos implicados en la dinámica de claros en los bosques y otros). En sistemas con disturbio crónico antrópico se ha encontrado que factores con pequeña influencia por separado pueden actuar de manera sinérgica (Martorell y Peters, 2005). El disturbio antrópico en los bosques de Chiapas incluye la tala selectiva e ilegal de árboles aislados para obtener madera de escaso valor comercial, la cosecha de ju veniles y ramas para leña, y el pastoreo extensivo esporádico (Barrón-Sevilla, 2002). Los efectos nocivos combinados del pisoteo, la herbivoría y la compactación del suelo sobre la regeneración de las especies arbóreas están bien identificados como un problema común de la restauración (Ramírez-Marcial et al., 1996, 2005). Por otra parte, la tala ocasional de árboles aislados y ramas modifi- 


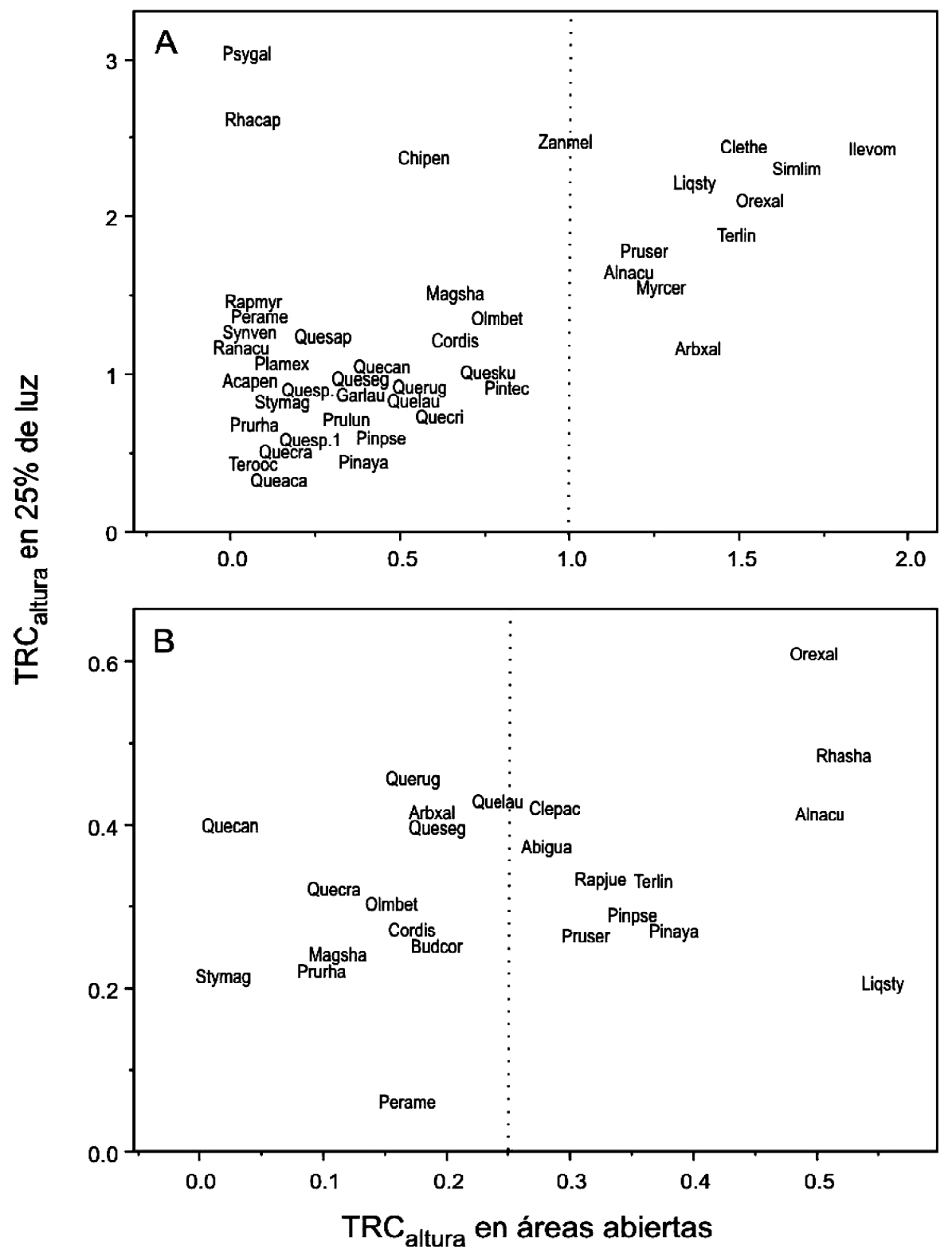

Figura 1. Relación entre las tasas relativas de crecimiento en altura (TRCaltura) bajo sombra (25\% de luz) respecto al desempeño bajo condiciones de radiación plena de plántulas de 42 especies arbóreas nativas en condiciones de jardín común o vivero (A) y para 24 especies evaluadas en condiciones de campo (B). Abigua = Abies guatemalensis Rehder, Acapen = Acacia pennatula $($ Schltdl. et Cham.) Benth., Alnacu = Alnus acuminata ssp. arguta (Schltdl.) Spach, Arbxal = Arbutus xalapensis Kunth, Budcor = Buddleja cordata Kunth, Chipen $=$ Chiranthodendron pentadactylon Larreat., Clepac $=$ Clethra pachecoana Standl. et Steyerm., Clethe $=$ Cleyera theoides Choisy, Cordis = Cornus disciflora DC., Garlau = Garrya laurifolia Hartw. ex Benth., Ilevom = Ilex vomitoria Aiton, Liqsty = Liquidambar styraciflua L., Magsha = Magnolia sharpii Miranda, Myrcer = Myrica cerifera L., Olmbet = Olmediella betschleriana (Göpp.) Loes., Orexal = Oreopanax xalapensis $($ Kunth) Decne. et Planch., Perame = Pe rsea americana Mill., Pin aya $=$ Pinus aya cahuite C.Ehrenb. ex Schltdl., Pinpse = Pinus pseudostrobus Lindl., Pintec $=$ Pinus tecunumanii Eguiluz et J.P.Perry, Plamex $=$ Platanus mexicana Moric., Prulun = Prunus lundelliana Standl., Prurha $=$ Prunus rhamnoides Koehne, Pruser $=$ Prunus serotina ssp. capuli (Cav.) McVaugh, Psygal = Psychotria galeottiana (M.Martens) C.M.Taylor et Lorence, Queaca = Quercus acatenangensis Trel., Quecan = Quercus candicans Née, Quecra $=$ Quercus crassifolia Humb. et Bonpl., Quecri $=$ Quercus crispipilis Trel., Quelau $=$ Quercus laurina Bonpl., Querug = Quercus rugosa Née, Quesap = Quercus sapotifolia Liebm., Queseg = Quercus segoviensis Liebm., Quesku = Quercus skutchii Trel., Quesp = Quercus sp 1., Ranacu $=$ Randia aculeata L., Rapjue $=$ Rapanea juergensenii Mez, $\mathbf{R}$ ap myr $=$ Rapanea myricoides (Schltdl.) Lundell, Rhacap = Rhamnus capreifolia var. grandifolia M.C.Johnst. et L.A.Johnst., Rhasha = Rhamnus sharpii M.C.Johnst. et L.A.Johnst., Simlim = Symplocos limoncillo Bonpl., Stymag = Styrax magnus Lundell, Synven = Synardisia venosa (Mast.) Lundell, Terlin = Ternstroemia lineata ssp. chalicophila $($ Loes.) B.M.Barthol., Terooc $=$ Ternstroemia oocarpa $($ Rose $)$ Melch. y Zanmel = Zanthoxylum melanostictum Schltdl. et Cham. 
Cuadro 2. Descripción de sitios de estudio, número de especies plantadas y duración de los ensayos de restauración forestal con especies arbóreas nativas en Los Altos y Montañas del Norte de Chiapas. Los hábitats incluidos como condición inicial son: $\mathrm{A}=$ área abierta, $\mathrm{M}=$ Matorral, $\mathrm{BT}=$ Bosque sucesional temprano, $\mathrm{BI}=$ Bosque sucesional intermedio, $\mathrm{BM}=$ Bosque maduro. $\mathrm{VIV}=$ tratamientos de sombra bajo condiciones de vivero: $0=\sin$ sombra, $1=50 \%, 2=75 \%$ y $3=90 \%$ de sombra.

\begin{tabular}{|c|c|c|c|c|c|}
\hline Sitios & $\begin{array}{l}\text { Altitud } \\
(\mathrm{m})\end{array}$ & $\begin{array}{l}\text { Precipitación } \\
\text { anual (mm) }\end{array}$ & $\begin{array}{l}\text { Condición } \\
\text { inicial }\end{array}$ & $\begin{array}{l}\text { Número de } \\
\text { especies }\end{array}$ & $\begin{array}{c}\text { Duración } \\
\text { (meses) }\end{array}$ \\
\hline Bazom 1 & 2,200 & 1,200 & $\mathrm{BT}, \mathrm{BM}$ & 9 & 60 \\
\hline Bazom 2 & 2,250 & 1,200 & BT & 11 & 60 \\
\hline Bazom 3 & 2,300 & 1,200 & BM & 5 & 48 \\
\hline Bazom 4 & 2,300 & 1,200 & BM & 5 & 72 \\
\hline Corazón de María & 2,380 & 1,100 & A, M & 4 & 120 \\
\hline Lindavista & 1,720 & 1,700 & A & 16 & 48 \\
\hline Huitepec & 2,500 & 1,300 & $\mathrm{~A}, \mathrm{BT}, \mathrm{BM}$ & 7 & 180 \\
\hline Moshviquil & 2,130 & 1,200 & A & 25 & 60 \\
\hline Mitzitón & 2,400 & 1,400 & $\mathrm{BT}, \mathrm{BI}$ & 9 & 60 \\
\hline ECOSUR & 2,120 & 1,200 & $\mathrm{VIV}=0,1,2,3$ & 42 & 12 \\
\hline San Cayetano & 1,620 & 1,800 & A & 15 & 12 \\
\hline La Trinitaria & 1,590 & 1,300 & A & 15 & 12 \\
\hline Montebello & 1,520 & 2,060 & $\mathrm{~A}, \mathrm{M}, \mathrm{BT}$ & 16 & 21 \\
\hline
\end{tabular}

ca la heterogeneidad lumínica en el interior de los bosques, con efectos sobre el crecimiento y la regeneración de especies arbóreas del interior (G. Méndez-Dewar, com. pers.), en escalas espaciales intermedias entre las reconocidas para los claros en el dosel del bosque (Denslow, 1987) y el moteado de luz o rayos de luz a través del dosel (sun flecks o lightflecks; Chazdon, 1988).

Autoecología y grupos funcionales. La condición necesaria para que las especies deseables puedan sobrevivir, establecerse y crecer en los sitios bajo restauración es, sin duda, que el microhábitat sea el apropiado (Ramírez-Marcial et al., 2006). Las condiciones de los sitios bajo restauración constituyen así filtros ambientales que determinan las "reglas de estucturación" de una comunidad vegetal (Temperton et al., 2004). La restauración de bosques tiene entre sus bases el conocimiento autoecológico de las especies clave y su agrupamiento en conjuntos con funciones similares en el sistema. El entendimiento de los procesos que guían el ciclo biológico y la dinámica poblacional es determinante para abordar los fenómenos relevantes en las escalas de acción de la restauración: el predio o parcela, el paisaje y la región.

Se han definido los requerimientos para la germinación de un conjunto amplio de especies (aproximadamente 140; Ramírez-Marcial et al., 2003). Para cada especie se han probado diversos métodos para estimular su germinación; una vez evaluados y afinados, éstos han permitido optimizar la producción de plántulas para la experimentación y el establecimiento de plantaciones diversificadas piloto.
Ante múltiples causas que limitan el establecimiento natural, como el pastoreo, el pisoteo y el ramoneo de plántulas y la tala selectiva en edades prerreproductivas, se ha optado por trabajar con un conjunto amplio de especies arbóreas de etapas sucesionales tempranas, intermedias y tardías. El conjunto de especies sobre el cual se ha obtenido más información relevante para su reintroducción en áreas degradadas incluye ocho gimnospermas, 11 especies de Quercus, 20 especies latifoliadas intolerantes a la sombra y 21 especies latifoliadas tolerantes a la sombra, pero intolerantes a la sequía prol on gada. Se han incluido especies bajo algún criterio de amenaza según la Unión Intemacional para la Conservación de la Nat u raleza (UICN), la Norma Oficial Mexicana NOM-059-ECOL-2001 (SEMARNAT, 2002), o ambas, como Abies guatemalensis, Acer negundo, Chiranthodendron pentadactylon, Magnolia sharpii, Pinus strobus var. chiapensis y Podocarpus matudai.

Durante los últimos 15 años se han establecido ensayos en el campo para evaluar la respuesta de un alto número de especies en una gama de condiciones ambientales (figura 1, cuadro 2). La duración de los ensayos fluctúa entre periodos relativamente cortos (1-3 años) hasta 15 años en un caso que aún se mantiene bajo evaluación (QuintanaAscencio et al., 2004). El número de especies utilizadas en cada localidad ha variado ampliamente (6-38 especies) en función de: (1) la disponibilidad del material biológico, (2) la disponibilidad de terreno y acceso, (3) el interés de los propietarios o ejidatarios por ciertas especies o algún grupo de ellas, y (4) el interés en preguntas particulares sobre el desempeño de las especies. 
Los resultados se han obtenido en tiempos y condiciones diversas. Por tanto, las conclusiones pueden ser limitadas en algún caso, pero cobran relevancia por tratarse de información de varios años respecto al desempeño de conjuntos diversos de especies. Las tasas relativas de crecimiento pueden ser hasta 4-5 veces mayores cuando los ensayos se realizan en jardín común o vivero respecto a las registradas para la misma especie en condiciones de campo -las condiciones reales en las que se efectúa la restauración(Ramírez-Marcial et al., 2006). Con base en los ensayos de campo, podemos proponer algunos grupos de especies con respuestas similares ante variables ambientales específicas. Por ejemplo, Oreopanax xalapensis, Rhamnus sharpii y Alnus acuminata ssp. arguta son fáciles de propagar por semilla y pueden establecerse relativamente bien en sitios abiertos, bajo arbustos como Baccharis vaccinioides (Ramírez-Marcial et al., 1996) e incluso en acahuales arbóreos. Especies como Pinus spp., Buddleja spp., Liquidambar styraciflua y Prunus serotina ssp. capuli son poco tolerantes a la sombra y se establecen con éxito en áreas abiertas; sus altas tasas de crecimiento les permiten formar en poco tiempo un dosel medianamente cerrado que promueve el establecimiento de otras especies tolerantes a la sombra pero intolerantes a la sequía prolongada, como Magnolia sharpii, Persea americana, Prunus lundelliana, P. rhamnoides y Styrax magnus, entre otras (figura 1). Este último grupo de especies del interior de bosques maduros probablemente tendrá menos posibilidades de mantener poblaciones viables bajo el actual patrón de disturbio de sus hábitats naturales. Su propagación y reintroducción podrían basarse en el enriquecimiento de áreas forestales florísticamente empobrecidas; dado que en estas áreas se mantienen usos tradicionales y potencialmente comerciales podría sustentarse la conservación de las especies en rodales maduros.

Patrones macroecológicos. La restauración de los ecosistemas forestales requiere de un marco teórico ecológico incluyente. Los fenómenos implicados cubren aspectos de la ecología de poblaciones, los ciclos biológicos y la tolerancia de las especies, hasta su distribución sobre gradientes ecológicos en escalas geográficas amplias (Huston y Smith, 1987; MacDonald et al., 2002; Hobbs, 2002). Los modelos más útiles pueden ser los más generales sobre los patrones y procesos implicados en la estructuración espacial y temporal de la biodiversidad, desde los niveles locales hasta los de paisaje y región (Storch et al., 2005). En la restauración práctica se buscaría sistematizar y predecir las respuestas de las especies individuales, y sus mezclas, en función de los gradientes ambientales presentes en una unidad del paisaje o en una región.

Con base en una hipótesis de Austin y Smith (1989), elaboramos un modelo sobre la variación espacial de la riqueza de especies arbóreas (figura 2). Se propone una bimodalidad de la diversidad a partir de las relaciones entre la disponibilidad de agua, la competencia interespecífica y la fertilidad o capacidad de los suelos para sustentar productividad primaria (González-Espinosa et al., 2004). En ambientes muy secos se esperaría una cubierta arbórea discontinua, con individuos relativamente espaciados entre sí y pertenecientes a un reducido grupo de especies (figura 2, I). En lugares con mayor disponibilidad de agua podría aumentar la diversidad debido a: (1) una mayor ocupación del espacio horizontal (un dosel forestal más cerrado), (2) una estructuración vertical en varios estratos, con especies heliófilas en el dosel y especies tolerantes a la sombra en uno o más estratos internos o inferiores, (3) ambas opciones simultáneamente (figura 2, II). En condiciones de mayor disponibilidad de humedad por mayor lluvia anual se verían favorecidas las especies con mayor capacidad competitiva y con mayor tolerancia a escasez de recursos. Como resultado, se esperarían bosques con una menor riqueza de especies (figura 2, III). Mayores cantidades de lluvia lixiviarían nutrimentos hacia las capas inferiores del suelo, donde sólo las especies con raíces profundas podrían aprovecharlos. La reducción de la calidad del sitio y el predominio de condiciones nutricionales marginales reduciría la capacidad competitiva y la densidad de unas pocas especies dominantes, al tiempo que se crearían oportunidades para un mayor número de especies, como en el modelo del disturbio moderado (Connell, 1978; figura 2, IV). Finalmente, el exceso de lavado de los suelos por niveles de precipitación pluvial excesivamente altos puede conducir a extremos de pobreza edáfica, e incluso ocurrencia

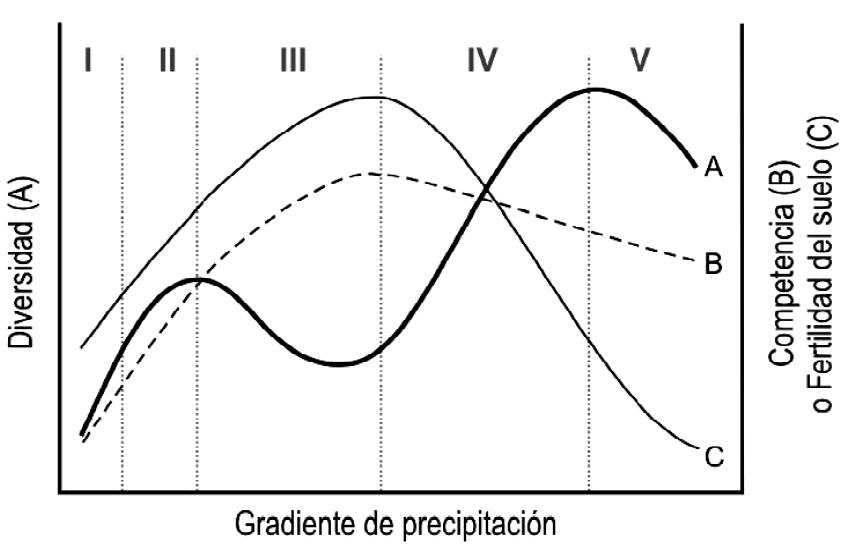

Figura 2. Relación hipotética entre la diversidad (o riqueza) de especies arbóreas (A) a lo largo de un gradiente de precipitación que interactúa con la intensidad de las relaciones competitivas (B) y la fertilidad (o) la calidad del suelo (C). Modificada a partir de Austin y Smith (1989). La curva de diversidad puede ser diferente en sus detalles, pero se propone su atributo de bimodalidad. En Chiapas se han encontrado los mayores valores de diversidad de árboles en la región de la Selva Lacandona, donde coinciden una alta precipitación y algunos de los suelos más pobres en todo el estado (González-Espinosa et al., 2004, 2005b). 
de condiciones de toxicidad por algunos compuestos poco solubles en condiciones muy ácidas, que solamente un pequeño grupo de especies puede tolerar en bosques de baja diversidad (figura 2, V).

Este modelo podría corresponder a un gradiente ambiental amplio, como el de las condiciones que prevalecen en los bosques de Chiapas. Su utilidad radica en su generalidad; busca predecir respuestas comunitarias sobre gradientes amplios. Sin embargo, el modelo es muy sencillo y no incluye varios factores asociados a la riqueza de especies como la naturaleza y propiedades de los suelos, o el escumimiento y la infil t ración del agua, que tendrían influencia de ámbito más local. Una limitante adicional es que dentro de la amplitud de las combinaciones de temperatura y humedad en Chiapas, desde el nivel del mar hasta cerca de 4,000 m de altitud, no se encuentran ambientes con menos de $800 \mathrm{~mm}$ de lluvia anual ni donde se excedan 4,700 mm (González-Espinosa et al., 2004, 2005b).

\section{Grupos académicos y la práctica de la restauración}

El proceso completo de la restauración de bosques y selvas no puede ser abordado en su totalidad por un grupo de investigación en las condiciones que privan en muchas comunidades indígenas. Se requiere de la colaboración del grupo con las instituciones y los grupos sociales. Los trabajos de restauración de bosques en los que se pasa de la escala de la parcela a las unidades del paisaje requieren de un importante esfuerzo de coordinación. En general, los grupos e instituciones académicas no tienen la capacidad de gestión para llevar a efecto proyectos de restauración forestal sobre territorios amplios. Ante las peculiares condiciones sociales, económicas y políticas que prevalecen en Chiapas, identificamos un modelo de vinculación y relaciones de apoyo financiero e intercambio de información del grupo de investigación con entidades de diferentes sectores (figura 3; Lyall et al., 2004).

En la actualidad, persiste una escasa o nula coordinación de las acciones relativas a los recursos forestales entre va rios actores: la planeación y el monitoreo locales pueden ser realizados por las organizaciones comunitarias, pero la responsabilidad de su planificación y ejecución a mayores escalas recae en las entidades de los gobiernos. Inicialmente nuestro grupo académico se ha restringido a la generación y uso del conocimiento para apoyar el desarrollo. Sin embargo, en años más recientes, mediante su diversificación y vinculación con organismos de la sociedad civil, ha establecido conexiones hasta ahora débiles o ausentes entre las comunidades y las dependencias encargadas del desarrollo; eventualmente, por su diversidad de habilidades, su experiencia acumulada y por la relativa estabilidad de sus dimensiones, podría incidir en el manejo adaptativo de la restauración (Guimerà et al., 2005).

Hay creciente interés en las comunidades indígenas por ejercer la autodeterminación dentro de sus territorios e involucrarse en la gestión de sus recursos (Bray y Merino, 2004; Cartagena-Ticona et al., 2005; Bojórquez-Vargas, 2006). Estas aspiraciones apuntan hacia una espera respetuosa de su propia motivación para definir y ejecutar la mayoría de las etapas del proceso de restauración. Por ahora parecen poco viables, al menos como un primer modelo de colaboración, las variantes del modelo de la investigación participativa, que generalmente presuponen acciones iniciales de parte del grupo académico y que requieren de propuestas técnicas de eficacia comprobada. Los procesos implicados en la restauración de bosques requieren que dos elementos fundamentales -las comunidades indígenas y el grupo académico-, definan y mantengan un conjunto mínimo de compromisos y prerrogativas con el cual podrían interactuar con las entidades de los gobiernos (cuadro 3). La indefinición o el incumplimiento de este tipo de acuerdos se encuentra en la base del fracaso de numerosos proyectos. De esta manera, por ejemplo, la experimentación requerida para sustentar responsablemente la restauración debe efectuarse en las condiciones de mayor seguridad y control sobre variables que puedan afectar los resultados. Ahora prevalecen en la región condiciones poco propicias para que las comunidades los alcancen y mantengan por el tiempo que sea necesario. Pocas excepciones lo confirman. En la práctica, para el grupo académico esto significa que antes que salir a las comunidades a ofrecer un posible apoyo, mejor se opte por mantener una amplia disposición para colaborar sólo con quienes se acerquen a solicitarlo de manera expresa y organizada.

Un grupo académico debe estar alerta a prestar su colaboración en los procesos de vinculación. Sin embargo, es necesario considerar que su acción efectiva en el proceso depende de una serie de elementos humanos y bases de información que requieren de mucho tiempo y dedicación para llegar a funcionar con eficiencia. Para ello, puede ser necesario privilegiar por un tiempo el uso de recursos hacia las tareas más especializadas que el grupo pueda hacer mejor en beneficio del colectivo más amplio al cual pertenece: investigación básica y aplicada dentro de estándares adecuados de calidad y eficiencia (figura 3). En este esquema el grupo académico puede enlazarse con ONGs en beneficio de las comunidades en la ejecución regional de proyectos para la restauración de bosques. Entre sus tareas estarían: (1) facilitar la participación de diversos grupos y entidades de gobierno implicados en el proceso (figura 3); (2) ofrecer elementos para la resolución de conflictos que pueden surgir entre elementos participantes; y (3) establecer y desarrollar sistemas de monitoreo de las prácticas de restauración. Estas actividades no las puede desarrollar el grupo académico con la flexibilidad financiera y operativa que le impone su nicho institucional tradicional. Cobra así relevancia su alianza o participación en organismos o fun- 
daciones sin fines de lucro que, emanando del ámbito académico y profesional, tengan capacidad para desarrollar $\mathrm{o}$ apoyar iniciativas empresariales comunitarias.

Las decisiones de las comunidades sobre el uso de sus recursos suelen cambiar debido a la frecuente carencia de modelos viables de desarrollo sustentable. Un grupo académico debe tomar conciencia de esta relativa indefinición de sus usuarios, de los procesos de ensayo y error de altemativas en los que a menudo se involucran, y abstenerse de pretender incidir en las decisiones que derivan en solicitudes de crédito y apoyo técnico por las comunidades. Mientras no se le invite a etapas más avanzadas y complejas del proceso adaptativo, el grupo debiera aplicarse en comprender los detalles del flujo de la información entre las comunidades de usuarios y difundir resultados útiles por medios audiovisuales como programas de radio y videos, ofreciendo la capacitación y asesoría subsecuentes. No es trivial este comentario, pues es común la asignación de recursos importantes a la producción de impresos y la promoción de la lectura en lenguas indígenas, cuando éstas están ciertamente vivas en las comınidades, pero de manera eminentemente oral.

La restauración es sólo una opción más para el uso sustentable del suelo que debe conciliarse con otras demandas sociales, con frecuencia conflictivas, como los sistemas productivos para el autoconsumo y el mercado y la asignación de territorios extensos para la conservación. La definición de las opciones demanda información sobre productividad y beneficios económicos y sociales incorporada en modelos de las estructuras y sus relaciones (Green et al., 2005). Por ahora no parece posible que se dispondrá en un plazo breve de la información detallada acerca de los atributos biológicos y consideraciones sociales y económicas para guiar con alto tino las prácticas de restauración de bosques y selvas en Chiapas. Ante esto, consideramos que los esfuerzos de investigación y desarrollo más redituables yacen en la construcción y validación de modelos con ensayos piloto, establecidos en una gran variedad de condiciones ecológicas y contextos socioeconómicos en los cuales haya sido invitado a colaborar el grupo académico.

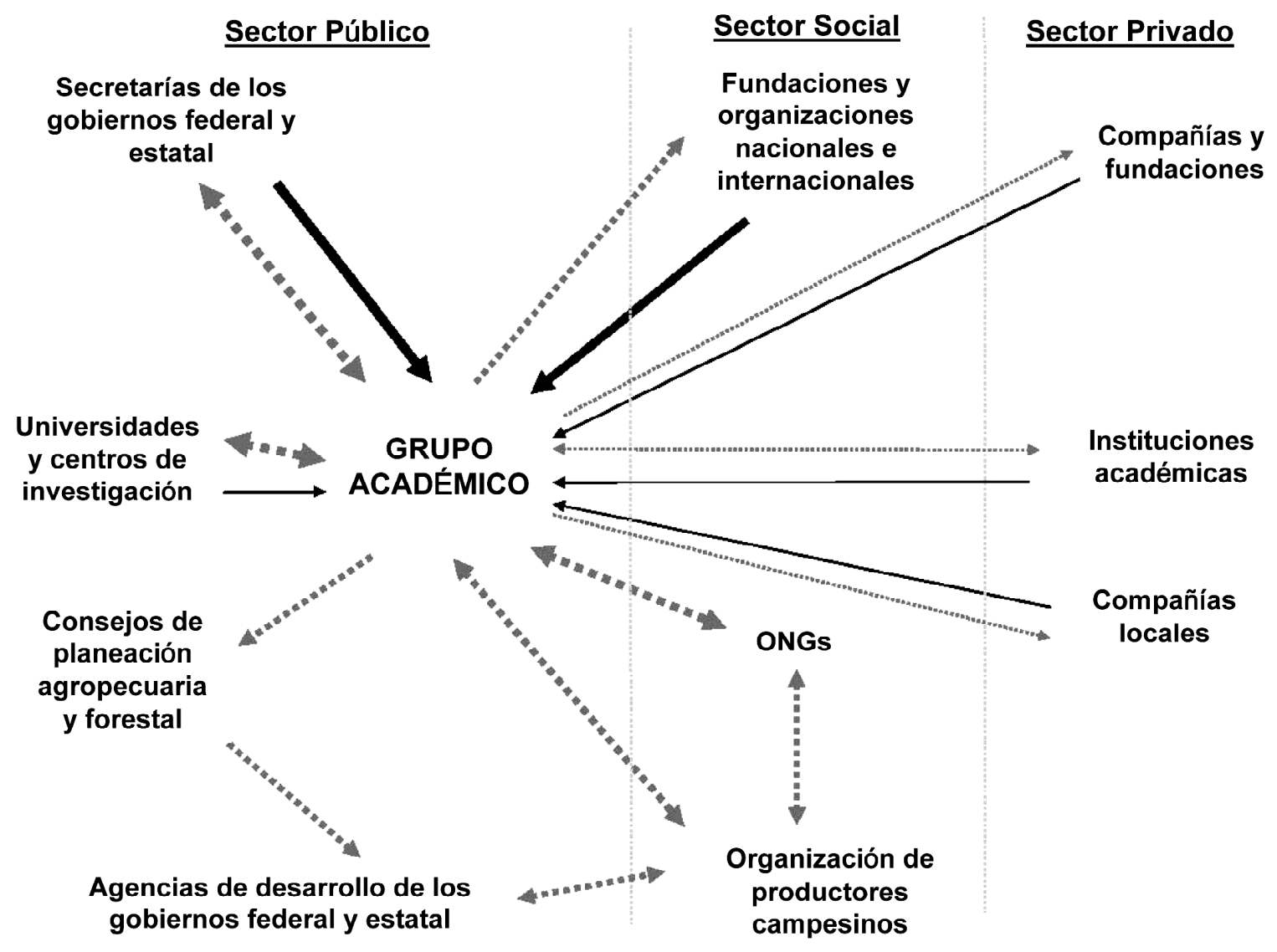

Figura 3. Relaciones actuales del grupo académico de ECOSUR con diferentes colaboradores y usuarios de la investigación aplicada a la restauración de bosques a través de acciones de vinculación. Las flechas continuas negras y su grosor indican flujos y monto relativo de financiamiento, respectivamente. Las líneas punteadas grises indican flujo e intensidad de información, respectivamente. Modificada a partir de Lyall et al. (2004). 
Cuadro 3. Propuesta de condiciones operativas deseables entre comunidades u organizaciones sociales y un grupo académico con capacidad para efectuar investigación básica y aplicada para definir y guiar acciones de restauración forestal en Chiapas.

Compromisos
Prerrogativas
Comunidades

Interés a largo plazo en el proceso y metas de la restauración de sus bosques.

Disponibilidad a largo plazo de las áreas necesarias para realizar las prácticas.

Disponibilidad para posponer y hacer respetar los acuerdos sobre aprovechamientos hasta el momento que se haya previsto en el plan de trabajo conjunto.

Grupo académico

Exploración y ofrecimiento de escenarios de manejo adaptativo para insertar los proyectos en los nichos de mercado emergentes.

Identificar o promover el desarrollo de interlocutores entre el grupo de investigación, las comunidades, otras organizaciones y diferentes entidades de gobierno que permitan una mayor estabilidad y viabilidad para el proceso.
Identificación y adopción de nichos de mercados potenciales para los productos de la restauración.

Autogestión para proponer modelos de organización propios.

Manejo técnico de la producción de las plantas; capacitación y supervisión sobre la calidad y variedad genética del material biológico utilizado.

Definición y supervisión de las prácticas, su evaluación y análisis-síntesis a distintos plazos.

\section{Agradecimientos}

Agradecemos a Francisco Javier Álvarez Sánchez y a Julia Carabias Lillo por invitarnos al simposio celebrado en Oaxaca en 2004. Durante años recibimos información y comentarios de M. Alcázar-Gómez, J. A. Barrón-Sevilla, L. Cayuela-Delgado, L. Galindo-Jaimes, D. Golicher, M. Huerta-Silva, S. Levy-Tacher, F. López-Barrera, L. Mascarúa-López, G. Méndez-Dewar, G. Montoya-Gómez, A. C. Newton y D. Torrez-Pérez. El apoyo de H. E. Castañeda Ocaña, P. Girón Hernández, M. Guillén Bautista, A. Luna Gómez y M. Martínez Icó ha sido clave en el campo, el laboratorio y el herbario. Dos árbitros anónimos y J.A. Meave contribuyeron sustancialmente a mejorar el texto. Esta investigación fue financiada por la Comisión Nacional para el Conocimiento y Uso de la Biodiversidad (CONABIO; F-019 y L-031), el Fondo Mexicano para la Conservación de la Naturaleza (A2-99006), los Fondos Mixtos (FOMIX-CHIS-2002-C01-4640 y FOMIX-CHIS-2005-C03-010), los Fondos Sectoriales (SEMARNAT-CONACYT C01-2002-048) y la Comisión de Comunidades Europeas a través de los proyectos BIOCORES (INCO Programme Framework 5, contrato No. ICA4-CT-2001-10095) y FOREST (Conservation and Restoration of Native Forests in Latin America, Alpha Project), coordinado por la Universidad de Alcalá (España).
Asbjornsen H., Vogt K.A. y Ashton M.S. 2004. Synergistic responses of oak, pine and shrub seedlings to edge environments and drought in a fragmented tropical highland oak forest, Oaxaca, Mexico. Forest Ecology and Management 192:313-334.

Austin M.P. y Smith T.M. 1989. A new model for the continuum concept. Vegetatio 83:35-47.

Barrón-Sevilla J.A. 2002. Efecto del disturbio antropogénico sobre la estructura y riqueza arbórea en bosques de pino-encino de Los Altos de Chiapas, México. Tesis de Maestría, El Colegio de la Frontera Sur, San Cristóbal de Las Casas, Chiapas, 25 pp.

Bautista-Cruz A., Gutiérrez-Castorena M.C., del Castillo-Sánchez R.F y Etchevers-Barra J.D. 2005. Cronosecuencia de un suelo y su clasificación en un área originalmente ocupada por bosque mesófilo de montaña. Terra Latinoamericana 23:147-157.

Berlin B., Breedlove D.E. y Raven P.H. 1974. Principles of Tzeltal Plant Classification: An Introduction to the Botanical Ethnography of a Mayan-Speaking People of the Highlands of Chiapas. Academic Press, Nueva York.

Bojórquez-Vargas A.R. 2006. Instituciones locales y apropiación social en la gestión de los recursos forestales bajo propiedad común. Estudio de caso de la comunidad agraria Teopisca, Chiapas, México. Tesis de Maestría, El Colegio de la Frontera Sur, San Cristóbal de Las Casas, Chiapas, 51 pp.

Bradshaw A.D. 1987. Restoration: an acid test for ecology. En: Jo rdan W.R., Gilpin M.E. y Aber J.D. Eds. Restoration Ecology: a Synthetic Approach to Ecological Research, pp. 2329, Cambridge University Press, Cambridge.

\section{Literatura citada}


Bradshaw A.D. 2002. Introduction and philosophy. En: Perrow M.R. y Davy A.J. Eds. Handbook of Ecological Restoration, Volume 1: Principles of Restoration, pp. 3-9, Cambridge University Press, Cambridge.

Bray D.B. y Merino L. 2004. La Experiencia de las Comunidades Forestales en México: Veinticinco Años de Silvicultura y Construcción de Empresas Forestales Comunitarias. Instituto Nacional de Ecología, Consejo Civil Mexicano para la Silvicultura Sostenible, A.C., México, D.F.

Breedlove D.E. 1981. Flora of Chiapas. Part I: Introduction to the Flora of Chiapas. California Academy of Sciences, San Francisco.

Breedlove D.E. 1986. Listados Florísticos de México IV. Flora de Chiapas. Instituto de Biología, Universidad Nacional Autónoma de México, México, D.F.

Cairns J. Jr. 2002. Rationale for restoration. En: Perrow M.R. y Davy A.J. Eds. Handbook of Ecological Restoration, Volume 1: Principles of Restoration, pp. 10-23, Cambridge University Press, Cambridge.

Camacho-Cruz A., González-Espinosa M., Wolf J.H.D. y de Jong B.H.J. 2000. Germination and survival of tree species in disturbed forests of the highlands of Chiapas, Mexico. Canadian Journal of Botany 78:1309-1318.

Cartagena-Ticona R.P., Parra-Vázquez M.R., Burguete-Cal y Mayor A. y López-Meza A. 2005. Participación social y toma de decisiones en los consejos municipales de desarrollo rural sustentable de Los Altos de Chiapas. Gestión y Política Pública 14:341-398.

Castillo A., Torres A., Velázquez A. y Bocco G. 2005. The use of ecological science by rural producers: a case study in Mexico. Ecological Applications 15:745-756.

Cayuela L., González M., Rey J.M., Ramírez N. y Martínez M. 2005. Imágenes de satélite revelan cómo desaparece el bosque en Chiapas. Quercus 232:60-61.

Cayuela L., Rey-Benayas J.M. y Echeverría C. 2006. Clearance and fragmentation of tropical montane forests in the Highlands of Chiapas, Mexico (1975-2000). Forest Ecology and Management 226:208-218.

Chazdon R.L. 1988. Sunflecks and their importance to forest understory plants. Advances in Ecological Research 18:2-54.

Collier G.A. 1976. Planos de Interacción del Mundo Tzotzil: Bases Ecológicas de la Tradición en Los Altos de Chiapas. Instituto Nacional Indigenista, México, D.F.

Collier G.A. y Quaratiello E.L. 1994. Basta! Land and the Zapatista Rebellion in Chiapas. The Institute for Food and Development Policy, Oakland.

Connell J.H. 1978. Diversity in tropical rain forests and coral reefs. Science 199:1302-1310.

De Jong B.H.J., Cairns M.A., Ramírez-Marcial N., Ochoa-Gaona S., Mendoza-Vega J., Haggerty P.K., González-Espinosa M. y March-Mifsut I. 1999. Land-use change and carbon flux between 1970s and 1990s in central highlands of Chiapas, Mexico. Environmental Management 23:373-385.

Denslow J.S. 1987. Tropical rain forest gaps and tree species diversity. Annual Review of Ecology and Systematics 18:431451.

Farjon A. y Styles B.T. 1997. Pinus (Pinaceae). Flora Neotropica, Monograph 75. The New York Botanical Garden, Nueva York.

Galindo-Jaimes L., González-Espinosa M., Quintana-Ascencio P. y García-Barrios L.E. 2002. Tree composition and structure in disturbed stands with varying dominance by Pinus spp. in the highlands of Chiapas, Mexico. Plant Ecology 62:259-272.

García-Barrios L.E. y González-Espinosa M. 2004. Change in oak to pine dominance in secondary forests may reduce shifting agriculture yields: experimental evidence from Chiapas, Mexico. Agriculture, Ecosystems and Environment 102:389401.

González-Espinosa M. 2005. Forest use and conservation implications of the Zapatista rebellion in Chiapas, Mexico. European Tropical Forest Research Network Newsletter 4344:74-76.

González-Espinosa M., Quintana-Ascencio P.F., Ramírez-Marcial N. y Gaytán-Guzmán P. 1991. Secondary succession in disturbed Pinus-Quercus forests of the highlands of Chiapas, México. Journal of Vegetation Science 2:351-360.

González-Espinosa M., Ochoa-Gaona S., Ramírez-Marcial N. y Quintana-Ascencio P.F. 1995. Current land-use trends and conservation of old-growth forest habitats in the highlands of Chiapas, Mexico. En: Wilson M.H. y Sader S.A. Eds. Conservation of Neotropical Migratory Birds in Mexico, pp. 190-198, Maine Agriculture and Forest Experiment Station, Miscellaneous Publication 727, Orono.

González-Espinosa M., Ramírez-Marcial N. y Ruiz-Montoya L. 2005a. Coords. Diversidad Biológica en Chiapas. Plaza y Valdés, Consejo de Ciencia y Tecnología del Estado de Chiapas, El Colegio de la Frontera Sur, México, D.F.

González-Espinosa M., Ramírez-Marcial N., Méndez-Dewar G., Galindo-Jaimes L. y Golicher D. 2005b. Riqueza de especies de árboles en Chiapas: variación espacial y dimensiones ambientales asociadas a nivel regional. En: González-Espinosa M., Ramírez-Marcial N. y Ruiz-Montoya L. Coords. Diversidad Biológica en Chiapas, pp. 81-125, Plaza y Valdés, Consejo de Ciencia y Tecnología del Estado de Chiapas, El Colegio de la Frontera Sur, México, D.F.

González-Espinosa M., Ramírez-Marcial N. y Galindo-Jaimes L. 2006. Secondary succession in montane pine-oak forests of Chiapas, México. En: Kappelle M. Ed. Ecology and Conservation of Neotropical Montane Oak Forests, Ecological Studies 185, pp. 209-221, Springer-Verlag, Berlín.

González-Espinosa M., Rey-Benayas J.M., Ramírez-Marcial N., Huston M.A. y Golicher D. 2004. Tree diversity in the northem Neotropics: regional patterns in highly diverse Chiapas, Mexico. Ecography 27:741-756.

Green R.E., Cornell S.J., Scharlemann J.P.W. y Balmford A. 2005. Farming and the fate of wild nature. Science 307:550-555.

Guimerà R., Uzzi B., Spiro J. y Nunes-Amaral L.A. 2005. Team assembly mechanisms determine collaboration network structure and team performance. Science 308:697-702.

Hellier A., Newton A.C. y Ochoa-Gaona S. 1999. Use of indigenous knowledge for rapidly assessing trends in biodiversity: a case study from Chiapas, Mexico. Biodiversity and Conservation 8:869-889.

Higgs E.S. 1997. What is good ecological restoration? Conservation Biology 11:338-348.

Hobbs R.J. 2002. The ecological context: a landscape perspective. En: Perrow M.R. y Davy A.J. Eds. Handbook of Ecological Restoration, Volume 1: Principles of Restoration, pp. 24-45, Cambridge University Press, Cambridge.

Holder C.D. 2004. Changes in structure and cover of a common property pine forest in Guatemala, 1954-1996. Environmental 
Conservation 31:22-29.

Huston M.A. y Smith T. 1987. Plant succession: life history and competition. The American Naturalist 130:168-198.

Ixtacuy-López O., Estrada-Lugo E. y Parra-Vázquez M.R. 2006. Organización social en la apropiación del territorio: Santa Marta Chenalhó, Chiapas. Relaciones 27:183-219.

Ixtacuy-López O. y Parra-Vázquez M. 2005. Cafeticultura y patrón de asentamiento en una comunidad india de Los Altos de Chiapas. En: Nazar A. Ed. Sociedad y Entorno en la Frontera Sur de México: Grupos Humanos, Ambiente y Políticas Públicas, pp. 129-167, El Colegio de la Frontera Sur, San Cristóbal de Las Casas, Chiapas.

Levy-Tacher S.I., Aguirre-Rivera J.R., Martínez-Romero M.M. y Durán-Fernández A. 2002. Caracterización del uso tradicional de la flora espontánea en la comunidad lacandona de Lacanhá, Chiapas, México. Interciencia 27:512-520.

López-Barrera F., Manson R.H., González-Espinosa M. y Newton A.C. 2006. Effects of the type of montane forest edge on oak seedling establishment along forest-edge-exterior gradients. Forest Ecology and Management 225:234-244.

López-Barrera F., Manson R.H., González-Espinosa M. y Newton A.C. 2007. Effects of varying forest edge permeability on seed dispersal in a neotropical montane forest. Landscape Ecology 22:189-203.

López-Barrera F., Newton A. y Manson R. 2005. Edge effects in a tropical montane forest mosaic: experimental tests of postdispersal acorn removal. Ecological Research 20:31-40.

Luna I., Velásquez A. y Velásquez E. 2001. México. En: Kappelle M. y Brown A.D. Eds. Bosques Nublados del Neotrópico, pp. 183-229, INBIO, Santo Domingo de Heredia.

Lyall C., Bruce A., Firn J., Firn M. y Tait J. 2004. Assessing enduse relevance of public sector research organisations. Research Policy 33:73-87.

MacDonald D.W., Moorhouse T.P. y Enck J.W. 2002. The ecological context: a species population perspective. En: Perrow M.R. y Davy A.J. Eds. Handbook of Ecological Restoration, Volume 1: Principles of Restoration, pp. 47-65, Cambridge University Press, Cambridge.

Mace R. y Pagel M. 1995. A latitudinal gradient in the density of human languages in North America. Proceedings of the Royal Society of London B 261:117-121.

Martorell C. y Peters E.M. 2005. The measurement of chronic disturbance and its effects on the threatened cactus Mammillaria pectinifera. Biological Conservation 124:199-207.

Miranda F. 1952. La Vegetación de Chiapas, Vol. 1. Ediciones del Gobierno del Estado, Tuxtla Gutiérrez.

Moore J.L., Manne L., Brooks T., Burgess N.D., Davies R., Rahbeck C., Williams P. y Balmford A. 2002. The distribution of cultural and biological diversity in Africa. Proceedings of the Royal Society of London B 269:1645-1653.

Montes-Avelar C.A. 2001. Patrones de diversidad florística en el paisaje agrícola de Los Altos de Chiapas, México. Tesis de Licenciatura (Biología), Facultad de Ciencias, Universidad Nacional Autónoma de México, México, D.F., 107 pp.

Montoya-Gómez G., Hernández-Ruiz F. y Mandujano-Granados M. 2003. Frontera Sur: de la riqueza de sus recursos naturales a la pobreza de sus habitantes. En: Montoya G., Bello E., Parra M. y Mariaca R. Eds. La Frontera Olvidada entre Chiapas y Quintana Roo, pp. 33-68, Consejo Estatal para la Cultura y las Artes de Chiapas, Tuxtla Gutiérrez.
Negrete-Yankelevich S. 2004. Integrating soil macroinvertebrate diversity, litter composition and secondary succession in a tropical montane cloud forest in Mexico. Tesis de Doctorado, Universidad de Edimburgo, Edimburgo, 345 pp.

Ochoa-Gaona S. y González-Espinosa M. 2000. Land-use and deforestation in the highlands of Chiapas, Mexico. Applied Geography 20:17-42.

Ochoa-Gaona S., González-Espinosa M., Meave J.A. y SoraniDal Bon V. 2004. Effect of forest fragmentation on the woody flora of the highlands of Chiapas, Mexico. Biodiversity and Conservation 13:867-884.

Palacio-Prieto J.L., Bocco G., Velázquez A., Mas J.-F., TakakiTakaki F., Victoria A., Luna-González L., Gómez-Rodríguez G., López-García J., Palma-Muñoz M., Trejo-Vázquez I., Peralta-Higuera A., Prado-Molina J., Rodríguez-Aguilar A., Mayorga-Saucedo R. y González-Medrano F. 2000. La condición actual de los recursos forestales en México: resultados del Inventario Forestal Nacional 2000. Investigaciones Geográficas 43:183-203.

Parra-Vázquez M.R. y Díaz-Hernández B.M. Eds. 1997. Los Altos de Chiapas: Agricultura y Crisis Rural. Tomo I, Los Recursos Naturales. El Colegio de la Frontera Sur, San Cristóbal de Las Casas, Chiapas.

Perales H.R., Benz B.F. y Brush S.B. 2005. Maize diversity and ethnolinguistic diversity in Chiapas, Mexico. Proceedings of the National Academy of Sciences of the USA 102:949-954.

Quintana-Ascencio P.F. y González-Espinosa M. 1993. Afinidad fitogeográfica y papel sucesional de la flora leñosa de los bosques de pino-encino de Los Altos de Chiapas, México. Acta Botanica Mexicana 21:43-57.

Quintana-Ascencio P.F., González-Espinosa M., Ramírez-Marcial N., Domínguez-Vázquez G. y Martínez-Icó M. 1996. Soil seed banks and regeneration of tropical rain forest from milpa fields at the Selva Lacandona, Chiapas, Mexico. Biotropica 28:192209.

Quintana-Ascencio P.F., Ramírez-Marcial N., González-Espinosa M. y Martínez-Icó M. 2004. Sapling survival and growth of conifer and broad-leaved trees in successional habitats in the highlands of Chiapas, Mexico. Applied Vegetation Science 7:81-88.

Ramírez-Marcial N. 2003. Survival and growth of tree seedlings in anthropogenically disturbed Mexican montane rain forests. Journal of Vegetation Science 14:881-890.

Ramírez-Marcial N., Camacho-Cruz A. y González-Espinosa M. 2003. Guía para la Propagación de Especies Leñosas Nativas de Los Altos y Montañas del Norte de Chiapas. El Colegio de la Frontera Sur, San Cristóbal de Las Casas.

Ramírez-Marcial N., Camacho-Cruz A. y González-Espinosa M. 2005. Potencial florístico para la restauración de bosques en Los Altos y las Montañas del Norte de Chiapas. En: GonzálezEspinosa M., Ramírez-Marcial N. y Ruiz-Montoya L. Coords. Diversidad Biológica en Chiapas, pp. 325-363, Plaza y Valdés, Consejo de Ciencia y Tecnología del Estado de Chiapas, El Colegio de la Frontera Sur, México, D.F.

Ramírez-Marcial N., Camacho-Cruz A., González-Espinosa M. y López-Barrera F. 2006. Establishment, survival and growth of tree seedlings under successional montane oak forests in Chiapas, Mexico. En: Kappelle M. Ed. Ecology and Conservation of Neotropical Montane Oak Forests, Ecological Studies 185, pp. 177-189, Springer-Verlag, Berlín. 
Ramírez-Marcial N., González-Espinosa M. y García-Moya E. 1996. Establecimiento de Pinus spp. y Quercus spp. en matorrales y pastizales de Los Altos de Chiapas. Agrociencia 30:249-257.

Ramírez-Marcial N., González-Espinosa M. y Williams-Linera G. 2001. Anthropogenic disturbance and tree diversity in montane rain forests in Chiapas, Mexico. Forest Ecology and Management 154:311-326.

Román-Cuesta R.M., Gracia M. y Retana J. 2003. Environmental and human factors influencing fire trends in ENSO and nonENSO years in tropical Mexico. Ecological Applications 13:1177-1192.

Romero-NájeraI. 2000. Estructura y condiciones microambientales en bosques pertubados de Los Altos de Chiapas, México. Tesis de Licenciatura (Biología), Facultad de Ciencias, Universidad Nacional Autónoma de México, México, D.F., 74 pp.

Rzedowski J. 1993. Diversity and origins of the phanerogamic flora of Mexico. En: Ramamoorthy T.P., Bye R., Lot A. y Fa J. Eds. Biological Diversity of Mexico: Origins and Distribution, pp. 129-144, Oxford University Press, Nueva York.

Sayer J., Chokkalingam U. y Poulsen J. 2004. The restoration of forest biodiversity and ecological values. Forest Ecology and Management 201:3-11.

SEMARNAT [Secretaría de Medio Ambiente y Recursos Naturales]. 2002. Norma Oficial Mexicana NOM-059-ECOL2001, Protección ambiental-Especies nativas de México de flora y fauna silvestres-Categorías de riesgo y especificaciones para su inclusión, exclusión o cambio-Lista de especies en riesgo. Diario Oficial de la Federación, miércoles 6 de marzo de 2002. México, D.F.

Storch P., Marquet P.A. y Gaston K.J. 2005. Untangling and entangled bank. Science 307:684-686.

Temperton V.M., Hobbs R.J., Nuttle T. y Halle S. 2004. Assembly Rules and Restoration Ecology: Bridging the Gap Between Theory and Practice. Island Press, Washington, D.C.

Vásquez-Sánchez M.A. Coord. 2003. Programa Estatal de Ordenamiento Territorial, Fases III y IV. El Colegio de la Frontera Sur, Gobierno de Chiapas, Tuxtla Gutiérrez.

Villafuerte-Solís D. 2004. La Frontera Sur de México: del TLC México-Centroamérica al Plan Puebla-Panamá. Plaza y Valdés, Instituto de Investigaciones Estéticas, Universidad Nacional Autónoma de México, México, D.F.

Villaseñor J.L. 2003. Dive rsidad y distribución de las Magnoliophyta de México. Interciencia 28:160-167.

Villaseñor J.L., Ibarra-Manríquez G., Meave J.A. y Ortíz E. 2005. Higher taxa as surrogates of plant biodiversity in a megadiverse country. Conservation Biology 19:232-238.

Wagner P.L. 1962. Natural and artificial zonation in a vegetation cover: Chiapas, Mexico. The Geographical Review 52:253274.

Wolf J. H.D. y Flamenco A. 2003. Patterns in species richness and distribution of vascular epiphytes in Chiapas, México. Journal of Biogeography 30:1689-1707.

Wolf J. H.D. y Flamenco A. 2005. Distribución y riqueza de epífitas de Chiapas. En: González-Espinosa M., Ramírez-Marcial N. y Ruiz-Montoya L. Coords. Dive rsidad Biológica en Chiapas, pp. 127-162, Plaza y Valdés, Consejo de Ciencia y Tecnología del Estado de Chiapas, El Colegio de la Frontera Sur, México, D.F.

Fecha de recepción: 24 de junio de 2005

Versión corregida: 28 de agosto de 2006

Aceptado: 28 de agosto de 2006 